\title{
DNA Methylation and Genetic Aberrations in Gastric Cancer
}

\author{
Genki Usuia,b Keisuke Matsusaka ${ }^{a, c}$ Yasunobu Mano ${ }^{a}$ Masayuki Urabe ${ }^{a, b}$ \\ Sayaka Funata $^{a}$ Masashi Fukayamab $^{b}$ Tetsuo Ushiku $^{b}$ Atsushi Kaneda $^{a}$ \\ aDepartment of Molecular Oncology, Graduate School of Medicine, Chiba University, Chiba, Japan; \\ bepartment of Pathology, Graduate School of Medicine, The University of Tokyo, Tokyo, Japan; \\ 'Department of Pathology, Chiba University Hospital, Chiba, Japan
}

\section{Keywords}

Gastric cancer - Epigenetics · DNA methylation .

Epstein-Barr virus · Helicobacter pylori

\begin{abstract}
Background: Gastric cancer (GC) is one of the leading causes of cancer-related deaths worldwide. GC is a pathologically and molecularly heterogeneous disease. DNA hypermethylation in promoter $\mathrm{CPG}$ islands causes silencing of tumorsuppressor genes and thus contributes to gastric carcinogenesis. In addition, various molecular aberrations, including aberrant chromatin structures, gene mutations, structural variants, and somatic copy number alterations, are involved in gastric carcinogenesis. Summary: Comprehensive DNA methylation analyses revealed multiple DNA methylation patterns in GCs and classified GC into distinct molecular subgroups: extremely high-methylation epigenotype uniquely observed in GC associated with Epstein-Barr virus (EBV), high-methylation epigenotype associated with microsatellite instability (MSI), and low-methylation epigenotype. In The Cancer Genome Atlas classification, EBV and MSI are extracted as independent subgroups of GC, whereas the remaining GCs are categorized into genomically stable
\end{abstract}

(GS) and chromosomal instability (CIN) subgroups. EBV-positive GC, exhibiting the most extreme DNA hypermethylation in the whole human malignancies, frequently shows CDKN2A silencing, PIK3CA mutations, PD- L1/2 overexpression, and lack of TP53 mutations. MSI, exhibiting high DNA methylation, often has $\mathrm{MLH} 1$ silencing and abundant gene mutations. GS is generally a diffuse-type $\mathrm{GC}$ and frequently shows $C D H 1 / R H O A$ mutations or CLDN18-ARHGAP fusion. $\mathrm{CIN}$ is generally an intestinal-type GC and frequently has TP53 mutations and genomic amplification of receptor tyrosine kinases. Key Messages: The frequency and targets of genetic aberrations vary depending on the epigenotype. Aberrations in the genome and epigenome are expected to synergistically interact and contribute to gastric carcinogenesis and comprehensive analyses of those in GCs may help elucidate the mechanism of carcinogenesis.

(c) 2020 S. Karger AG, Basel

\section{Introduction}

Gastric cancer (GC) is the third leading cause of cancer death worldwide [1]. GC is a histologically heterogeneous cancer. Most GCs are adenocarcinomas and are classified karger@karger.com

www.karger.com/dig

Karger

(C) 2020 S. Karger AG, Basel

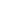


into 2 main histological types: intestinal type and diffuse type, according to the Lauren classification [2]. These subtypes show biological differences in regional incidence, clinical features, and prognosis [3]. In the World Health Organization classification, GCs are classified into some subtypes: tubular, papillary, mucinous, poorly cohesive including signet ring cells and other subtypes, and mixed adenocarcinomas [4]. Furthermore, immunohistochemistry has already shown that GC includes adenocarcinoma with a gastric phenotype and also identified new subtypes of GC, including adenocarcinoma with differentiation of the fundic gland [5].

A variety of environmental factors are involved in developing GC, including smoking, alcohol, diet, and Helicobacter pylori (H. pylori) infection [3]. H. pylori is an important risk factor for $\mathrm{GC}$, and a previous prospective study revealed that subjects infected with $H$. pylori had a high incidence of GC [6]. Most H. pylori-related GCs occur in the distal stomach and usually demonstrate intestinal-type histology [4]. Epstein-Barr virus (EBV), the well-known human herpes virus usually acquired during childhood, also contributes to gastric carcinogenesis [7]. EBV(+) GCs account for 7-15\% of GCs and show characteristic clinical features such as proximal location of the stomach and relatively better prognosis [7]. EBV(+) GCs histologically demonstrate irregular sheets, trabecula, illdefined tubes or syncytia of polygonal cells with dense infiltration of lymphocytes and are referred to as gastric carcinoma with lymphoid stroma [4].

Various molecular aberrations are involved in gastric carcinogenesis. Epigenetic aberrations, such as DNA methylation in the promoter $\mathrm{CpG}$ island, are involved in the development of GC $[8,9]$. Aberrant methylation of tumor-suppressor genes causes transcriptional silencing, leading to carcinogenesis. Previous studies revealed that GCs were classified into at least 2 DNA hypermethylation subtypes with a high or low frequency of aberrant methylation at the $\mathrm{CpG}$ islands in the genome or in the $5^{\prime}$ regions of genes $[10,11]$. Additional studies reported that the frequency of DNA methylation in the promoter region was higher in $\mathrm{EBV}(+)$ GCs than other GCs, although the number of analyzed cancer-associated genes was limited $[7,12]$. A comprehensive analysis with Illumina's Infinium BeadArray identified distinct DNA methylation patterns in GCs and classified GCs into 3 epigenotypes: extremely high-methylation epigenotype (E-HME), high-methylation epigenotype (HME), and low-methylation epigenotype (LME) [13]. E-HME is the unique phenotype of $\mathrm{EBV}(+) \mathrm{GCs}$, exhibiting aberrant hypermethylation of hundreds of genes specifically observed in this subgroup, such as CDKN2A (p16INK4A). HME shows aberrant methylation of genes more frequently than LME; MLH1 promoter hypermethylation is frequently observed in HME.

Three-dimensional chromatin organization is also involved in the development of GC. Aberrant chromatin structures are associated with the regulation of gene expression, and these chromatin structures are detected in cancer $[14,15]$. Recent studies revealed long-range chromatin interaction in regions related to genomic rearrangements [16]. Genomic rearrangements are involved in "enhancer hijacking," promoting the expression of CCNE1 and IGF2. Our latest study of EBV(+) GC has shown that episomal viral genomes interact with human genome and rewire the human chromatin structure [17]. Silenced enhancers are aberrantly activated by the chromatin rewiring (named "enhancer infestation") and can upregulate neighboring proto-oncogenes to promote gastric carcinogenesis.

Genetic aberrations also contribute to gastric carcinogenesis [18]. TP53 gene mutations are the most frequent genetic aberrations in GCs [19]. TP53 gene mutations are found in about half the cases of GCs. Other mutations are observed in several genes, including CDH1, ARID1A, KRAS, PIK3CA, APC, CTNNB1, and SMAD4 [20]. In addition, RHOA mutations are detected in some cases of diffuse-type GCs [21, 22]. In 2014, The Cancer Genome Atlas (TCGA) proposed a molecular classification of GCs [23]. In this classification, EBV(+) GC exhibiting extremely high methylation uniquely, and microsatellite instability (MSI) involving high methylation, for example, $M L H 1$, are extracted as independent subgroups of GC. The remaining GCs, thus mostly exhibiting LME, are categorized into 2 subtypes according to the somatic copy number alterations (sCNAs): chromosomal instability (CIN) and genomically stable (GS) subgroups. This molecular classification has now been widely known throughout the world. In this study, we will review the classification of GC into molecular subtypes, especially by DNA methylation and genetic aberrations.

\section{Epigenetic Aberrant in Gastric Cancer}

\section{DNA Methylation and Gastric Carcinogenesis}

DNA methylation is the common epigenetic aberration. The methylation of 5-position of cytosine bases at CpG dinucleotides such as 5-methylcytosine is well known $[8,9]$. CpG islands in promoter regions are basically unmethylated, and expression of the gene is regu-
Usui/Matsusaka/Mano/Urabe/Funata/ Fukayama/Ushiku/Kaneda 
lated by histone modification. Aberrant methylation of promoter $\mathrm{CpG}$ islands causes the transcriptional silencing, leading to the expression of the downstream gene. The silencing of tumor-suppressor genes caused by methylation is one of the major mechanisms of carcinogenesis $[8,9,18]$.

The level of DNA methylation is affected by environmental factors. Previous studies showed that aging and smoking are involved in the accumulation of aberrant methylation in CpG islands [24]. In addition, chronic inflammation is known to accelerate the accumulation of aberrant methylation in noncancerous tissues. For example, ulcerative colitis, chronic hepatitis, and reflux esophagitis cause the inflammation, leading to aberrant $\mathrm{CpG}$ island methylation [7]. This aberrant CpG island methylation of these noncancerous tissues is considered to associate with carcinogenesis. Indeed, the mouse colitis model induced by dextran sodium sulfate revealed that DNA methylation of CpG island was accumulated in colonic mucosa [25].

Chronic inflammation caused by $H$. pylori is associated with gastric carcinogenesis [7]. H. pylori infection causes aberrant promoter methylation, silencing the expression of tumor-suppressor genes such as RUNX3, $L O X$, and $C D H 1$. Previous studies suggested that chronic inflammation caused by $H$. pylori infection may induce aberrant DNA methylation rather than any factor of $H$. pylori itself [26]. Another study using a Mongolian gerbil model supported that both $H$. pylori infection itself and aberrant methylation in gastric mucosa contribute to $H$. pylori-related gastric carcinogenesis [27].

\section{Epigenetic Aberrant and Carcinogenesis in EBV(+) \\ Gastric Cancer}

EBV infection is associated with epigenetic aberrant and gastric carcinogenesis [28]. Comprehensive DNA methylation analyses showed that EBV infection could cause de novo DNA methylation in an LME GC cell, MKN7 [13]. Furthermore, EBV infection also induced aberrant DNA methylation within a month in an immortalized normal gastric epithelial cell line, GES1 [29]. The mechanism of epigenetic aberration and gastric carcinogenesis during EBV infection has not been completely resolved; however, repression of TET2, a resistance factor against DNA methylation, is involved in DNA methylation [30].

Moreover, not only DNA methylation but also other epigenetic alterations caused by EBV infection may contribute to carcinogenesis. EBV infection causes enhancer activation and repression, which may be associated with gastric carcinogenesis [31]. A recent study suggested that enhancer activation through ATF3 might be involved in carcinogenesis [32]. Our latest study has revealed that interaction of episomal EBV genome may directly rewire the host chromatin structure to aberrantly activate protooncogenes and promote gastric carcinogenesis [17]. In the study, Hi-C analysis showed the specific interaction between viral and human genomes in $\mathrm{EBV}(+) \mathrm{GC}$ cell lines and primary tissues of human $\mathrm{EBV}(+) \mathrm{GC}$. The human genomic regions interacting with the EBV genome were named as EBVIRs (EBV-interacting regions), and defined by high-resolution technique using $4 \mathrm{C}$-seq. EBVIRs were found to be modified bivalently with active histone marks such as $\mathrm{H} 3 \mathrm{~K} 4 \mathrm{me} 1$ and $\mathrm{H} 3 \mathrm{~K} 27 \mathrm{ac}$ and repressive histone mark $\mathrm{H} 3 \mathrm{~K} 9 \mathrm{me}$. This is because EBV genome preferentially interacts with $\mathrm{H} 3 \mathrm{~K} 9 \mathrm{me} 3(+)$ heterochromatins and converts the regions from repressive $\mathrm{H} 3 \mathrm{~K} 9 \mathrm{me} 3(+)$ state to active $\mathrm{H} 3 \mathrm{~K} 4 \mathrm{me} 1(+) / \mathrm{H} 3 \mathrm{~K} 27 \mathrm{ac}(+)$ state. Enhancers silenced in the heterochromatin are thus aberrantly activated in EBVIRs and can upregulate nearby GC-related genes such as TGFBR2 and MZT1 [17].

\section{Accumulation of DNA Methylation and Risk of Developing GC}

Epigenomic aberrations accumulated in apparently normal tissues reportedly modify tumor risk and are thus suggested to be utilized as tumor risk markers [33, 34]. In noncancerous gastric mucosa with $H$. pylori infection, both driver genes and passenger genes have been methylated [35]. Some passenger genes are more highly methylated in gastric mucosa of subjects with GC than those without GC. This result suggests that the methylation level of particular passenger genes may help predict the risk of developing GC. A recent study also suggested that the methylation levels of gastric mucosal may be useful to predict the risk of developing GC [36]. Indeed, a prospective clinical study revealed that patients with high methylation of marker genes showed a higher incidence of metachronous GC $[37,38]$. However, the marker gene that reflects the risk of primary GC has not yet been established.

It also remains unclear how promoter regions of each gene are methylated after $H$. pylori infection. A previous study identified DNA methylation-sensitive genes and DNA methylation-resistant genes [39]. DNA methylation-sensitive genes, including CDKN2A, are methylated in the whole promoter regions by EBV infection, whereas DNA methylation-resistant genes methylated only in the surrounding of promoter regions. Besides, a comprehensive DNA methylation analysis using GES1 with EBV in- 


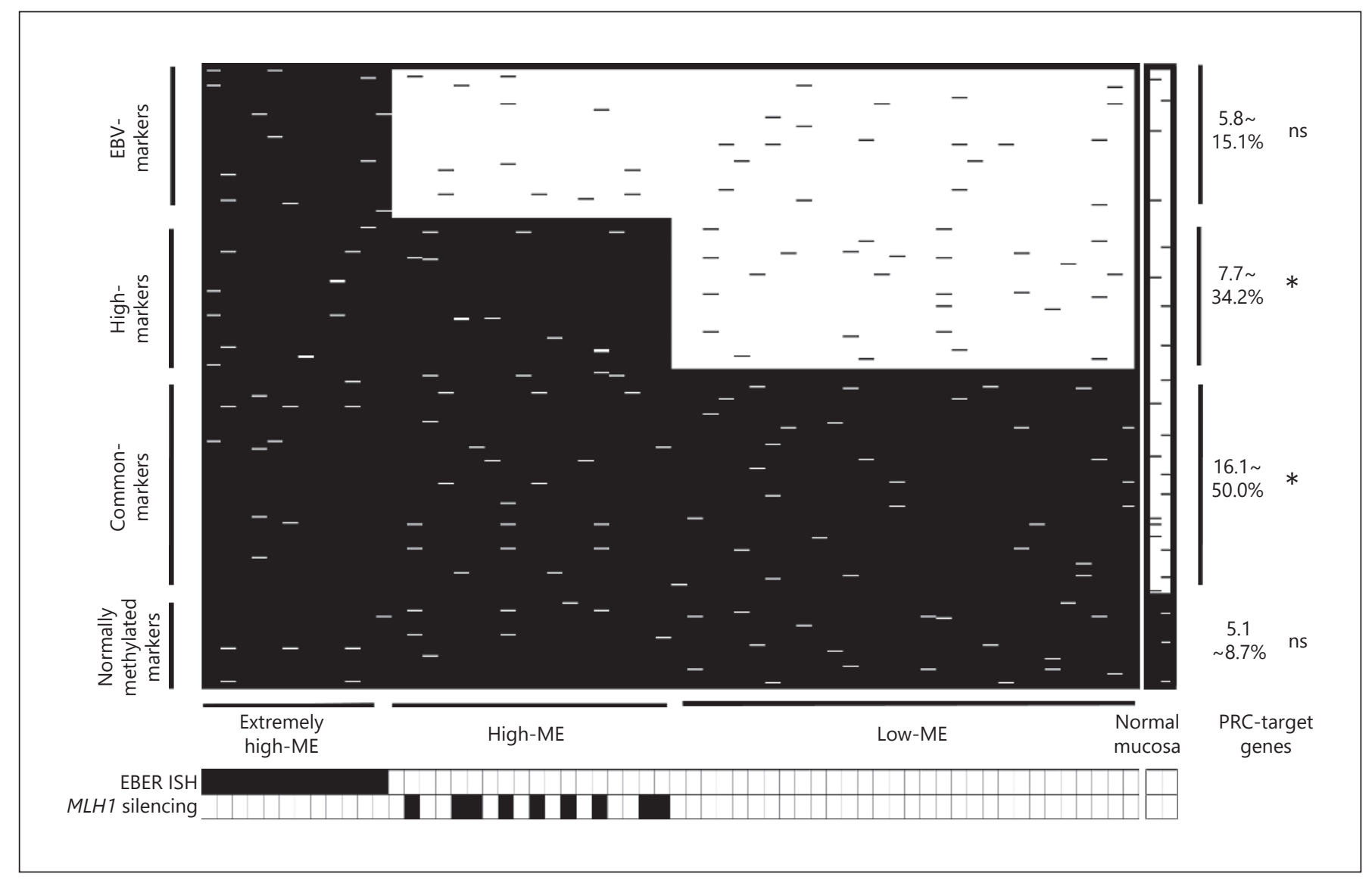

Fig. 1. Distribution of DNA hypermethylation of marker genes in epigenotypes. GC is classified into distinct molecular subgroups: E-HME, HME, and LME. In E-HME, all GCs are positive for EBER in situ hybridization. In HME, MLH1 is frequently silenced. In LME, all GCs are negative for EBER in situ hybridization and lack MLH1 silencing. The frequency of PRC-target genes is larger in high and common markers than in all genes. PRC-target genes in

fection revealed that alternant DNA methylation was acquired from $\mathrm{CpG}$ peripheral regions to core regions [29]. Although the process of acquiring DNA methylation in cell lines has been reported, further studies are necessary to be conducted to clarify whether these dynamic changes in methylation status are also confirmed in clinical samples of the non-neoplastic gastric mucosa.

\section{Methylation of Target Genes in Each Epigenotype}

GCs are subdivided into subgroups according to aberrant DNA methylation: E-HME, HME, and LME (Fig. 1) [13]. E-HME is uniquely observed in GC associated with $\mathrm{EBV}(+)$ GCs. This epigenotype has specifically methylated genes, including $C D K N 2$, which are unmethylated in other epigenotypes. HME is associated with MSI and has embryonic stem cells are enriched in both high and common markers $\left(^{*}\right)$ but not significant in EBV markers and normally methylated markers (NS). E-HME, extremely high-methylation epigenotype; HME, high-methylation epigenotype; LME, lowmethylation epigenotype; GC, gastric cancer; PRC, polycomb repressive complex; EBV, Epstein-Barr virus. frequent methylation of $M L H 1$ (46\%). HME has methylated genes that are unmethylated in LME. Interestingly, these methylated genes are also methylated in E-HME, except for MLH1 [13]. LME has methylated genes that are unmethylated in normal gastric mucosa. These methylated genes are also methylated in other epigenotypes.

A comprehensive analysis of DNA methylation in GC has proposed 3 subtypes of marker genes identifying each epigenotype [13]. EBV markers are specifically methylated only in E-HME. High markers are methylated in both E-HME and HME, but not methylated in LME. Common markers are methylated in all epigenotypes; however, are unmethylated in normal gastric mucosa. The frequency of polycomb repressive complex (PRC)target genes is lower in EBV markers than in both high 
Table 1. Methylation marker genes and enriched gene ontology terms

Enriched GO terms

Included methylated genes

EBV markers

Chemotaxis

Regulation of cellular response to growth factor stimulus

Cell surface receptor signaling pathway involved in cell-cell signaling

Developmental growth

BMP4, EFNA5, and TGFB2

TGFB1I1, PELO, and SEMA6A

BMP4, TGFB1I1, and LGR6

TGFB2, EFNA5, and FBLN5

High markers

Cell morphogenesis involved in differentiation

FBLN1, NKX6-1, and THY1

Synaptic signaling

Embryonic morphogenesis

EGR2, PTPRD, and WNT7A

FOXL2, GATA3, and RET

Synapse organization

PTPRD, WNT7A, and TMEFF1

Common markers

Axon development

Synapse organization

Cell-adhesion molecules

Regulation of neuron differentiation

\author{
PTPRM, SLIT3, UNC5C, and EPHA6 \\ $A D G R B 3, P O U 4 F 1$, and TMEFF2 \\ CDH4, NCAM1, NCAM2, and JAM2 \\ PTPRO, SFRP1, RGMA, and MIR137
}

Three groups of methylation marker genes have been reported to determine methylation epigenotypes of GC [29]. The GO analysis using reported markers has identified significantly enriched GO terms in marker genes. Representative enriched GO terms are listed in the table. These GO terms include several methylated genes which are considered to function as tumor-suppressor genes. GO, gene ontology; $E B V$, Epstein-Barr virus; GC, gastric cancer.

and common markers. These differences in frequency of PRC-target genes suggest that the methylation mechanism in E-HME is different from other epigenotypes [13, 28]. Another comprehensive analysis of DNA methylation in both GES1/MKN7 and clinical GC samples also extracted 3 subtypes of marker genes that identify each epigenotype [29]. Even with these extracted markers, the frequency of PRC-target genes in the EBV markers differs from that in other markers.

The gene ontology (GO) terms enriched in the reported EBV markers [29] are summarized in Table 1: chemotaxis (e.g., BMP4, EFNA5, and TGFB2), regulation of cellular response to growth factor stimulus (e.g., TGFB1I1, PELO, and SEMA6A), cell surface receptor signaling pathway involved in cell-cell signaling (e.g., BMP4, TGF$B 1 I 1$, and $L G R 6$ ), and developmental growth (e.g., TGFB2, EFNA5, and FBLN5). High markers show enrichment of GO terms such as cell morphogenesis involved in differentiation (e.g., FBLN1, NKX6-1, and THY1), synaptic signaling (e.g., EGR2, PTPRD, and WNT7A), embryonic morphogenesis (e.g., FOXL2, GATA3, and RET), and synapse organization (e.g., PTPRD, WNT7A, and TMEFF1). Common markers show enrichment of GO terms such as axon development (e.g., PTPRM, SLIT3, UNC5C, and EPHA6), synapse organization (e.g., ADGRB3, POU4F1, and TMEFF2), cell-adhesion mole- cules (e.g., CDH4, NCAM1, NCAM2, and JAM2), and regulation of neuron differentiation (e.g., PTPRO, SFRP1, $R G M A$, and MIR137). Multiple tumor-suppressor genes are included in these categories, and their silencing by DNA methylation might be associated with gastric carcinogenesis of each epigenotype.

TCGA classification stratified LME into GS and CIN subgroups. Although GS and CIN are indistinguishable by DNA methylation, their histological and genetic findings are different and will be discussed in the next chapter.

\section{Genetic Aberrations in Gastric Cancer}

\section{Genetic Mutations}

Genetic mutation is one of the most common mechanisms of carcinogenesis [18]. HME has especially frequent mutations, including TP53, PIK3CA, KRAS, ARID1A, PTEN, ERBB3, and HLA-B [23]. In this subtype, histocompatibility complex class I genes, including $B 2 M$ and $H L A-B$, are frequently mutated. These mutations may work better in hypermutated GCs by suppressing the immune response [23].

Some of these genes are also mutated in other epigenotypes, such as TP53, ARID1A, KRAS, and PIK3CA [23]. TP53 mutation is the most frequent genetic aberration 
Fig. 2. Molecular aberrations in epigenotypes. E-HME is observed in EBV-positive GC; frequently have CDKN2A methylation, PIK3CA mutations, and PD-L1/2 overexpression; and generally lack TP53 mutations. Proto-oncogenes such as MZT1 are activated by "enhancer infestation" [17]. HME often has $M L H$ methylation and abundant gene mutations, including $P I K$ 3CA, ARID1A, and RTK. LME contains GS and CIN subtypes. The GS subtype frequently has $C D H 1 / R H O A$ mutations or CLDN18-ARHGAP fusion. The CIN subtype has genomic amplification of RTKs and frequently has TP53 mutations. In each column, open and closed circles show unmethylated and hypermethylated regions, and the strikethrough shows gene mutation. E-HME, extremely high-methylation epigenotype; HME, high-methylation epigenotype; LME, low-methylation epigenotype; RTK, receptor tyrosine kinase; GS, genomically stable; CIN, chromosomal instability. MSI, microsatellite instability; EBV, Epstein-Barr virus; H. pylori, Helicobacter pylori.

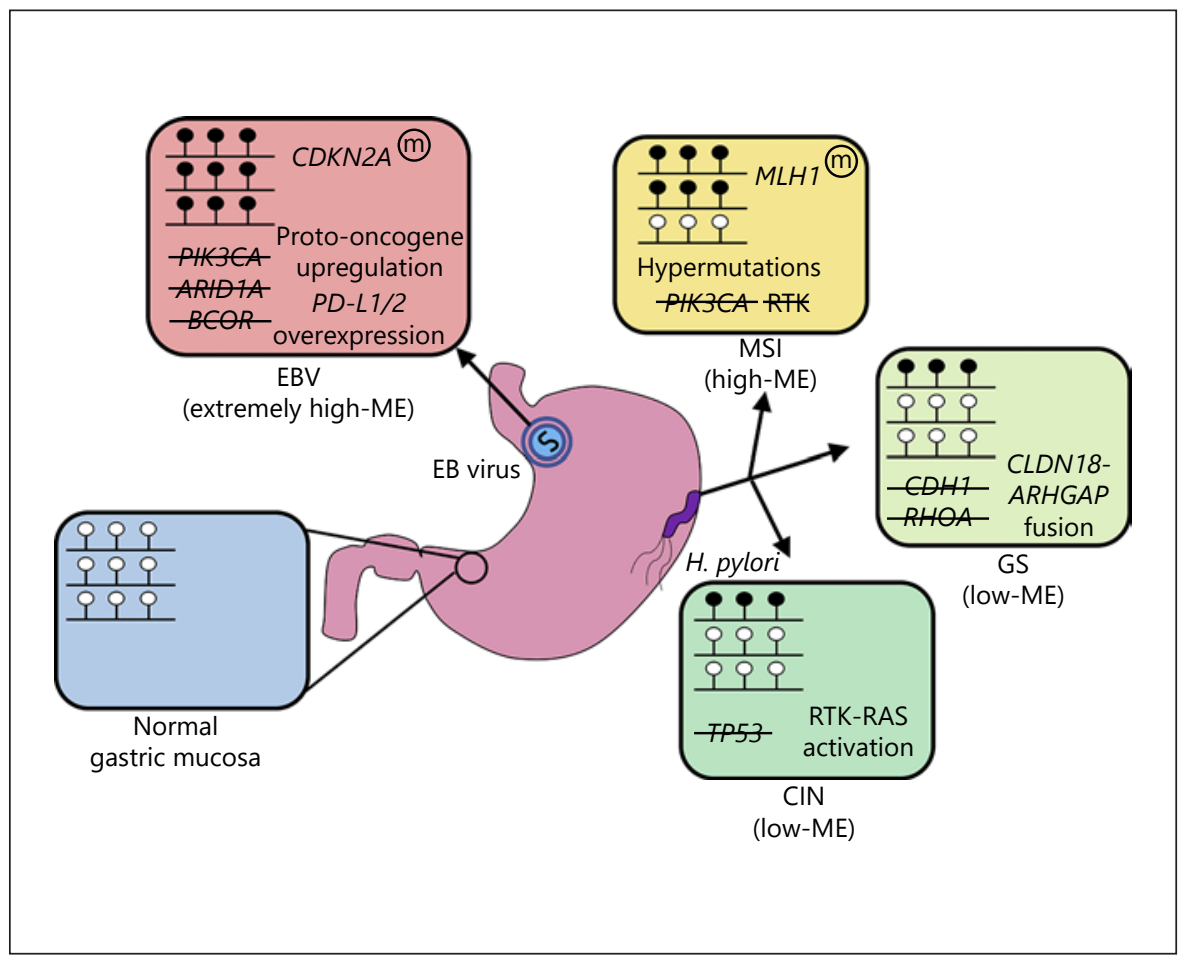

Table 2. Characteristic histology and major molecular aberrations in each epigenotype

\begin{tabular}{|c|c|c|c|c|}
\hline E-HME & Gastric carcinoma with lymphoid stroma & CDKN2A & $\begin{array}{l}\text { PIK3CA } \\
\text { ARID1A } \\
B C O R\end{array}$ & $\begin{array}{l}\text { Latent EBV infection } \\
\text { Enhancer infestation } \\
P D-L 1 / 2 \text { overexpression }\end{array}$ \\
\hline HME & None & $M L H 1$ & $\begin{array}{l}\text { PIK3CA } \\
\text { ARID1A } \\
\text { RTK }\end{array}$ & \\
\hline \multicolumn{5}{|l|}{ LME } \\
\hline GS & Diffuse type & & $\begin{array}{l}C D H 1 \\
R H O A\end{array}$ & CLDN18-ARHGAP fusion \\
\hline CIN & Intestinal type & & TP53 & RTK-RAS activation \\
\hline
\end{tabular}

The histology is different among molecular subtypes [4]. In particular, E-HME demonstrates characteristic histology referring to as gastric carcinoma with lymphoid stroma. Gene mutations, structural variants, and sCNAs are also different among the subtypes [23]. E-HME, extremely high-methylation epigenotype; HME, high-methylation epigenotype; LME, low-methylation epigenotype; EBV, Epstein-Barr virus; RTK, receptor tyrosine kinase; GS, genomically stable; CIN, chromosomal instability; sCNAs, somatic copy number alterations.

among several driver genes of GCs [19]. p53 contributes to DNA repair, cell cycle control, and programmed cell death [40]. TP53 mutations have been identified in both intestinal type and diffuse type and are detected in $71 \%$ of the CIN subtype [23]. Almost all EBV(+) GCs lack TP53 mutation; however, PIK3CA, ARID1A, and BCOR mutations are frequently observed in $\mathrm{EBV}(+) \mathrm{GCs}$. RHOA mutations are frequently observed in diffuse type/GS subtype. Because patients with diffuse-type GCs have a poor prognosis, $R H O A$ mutation can be a clinically significant 
target for treatment. $\mathrm{CDH} 1$ germline mutation is widely known to be associated with hereditary diffuse gastric cancer. $\mathrm{CDH} 1$ somatic mutation is frequently detected in diffuse type/GS subtype. TCGA study reported that $37 \%$ of the GS subtype has the $C D H 1$ somatic mutation [23].

\section{sCNAs and Structural Variants}

sCNAs and structural rearrangements also play an important role in cancer development through the selective activation or inactivation of oncogenes and tumor-suppressor genes [23]. The most frequently amplified genes in GC are associated with receptor tyrosine kinase (RTK)/ RAS/mitogen-activated protein kinase (MAPK) signaling such as HER2, EGFR, MET, FGFR2, and RAS [3, 23]. There may be RTK/RAS/MAPK-related amplifications in $30-40 \%$ of GCs. In these GCs, therapeutic effects targeting RTK/RAS/MAPK-related signal transduction are expected. Additionally, amplifications of JAK2, CD274 $(P D-L 1)$, and PDCD1LG2 (PD-L2) are frequently observed in EBV(+) GCs [23]. These aberrant amplifications can be a target for immunotherapy.

TCGA study reported the CLDN18-ARHGAP26 fusions in diffuse type/GS subtype [23]. ARHGAP26 is associated with the Rho-effector pathway. The CLDN18ARHGAP fusions are enriched in GS subtype and exclusively exist with both $R H O A$ and $C D H 1$ mutations. This exclusivity suggests that both $C D H 1 / R H O A$ mutations and CLDN18-ARHGAP fusion contribute to carcinogenesis depending on cell adhesion-related Rho signaling in this subtype $[3,23]$.

\section{Summary of Histology and Genetic Aberrations in \\ Each Epigenotype}

Various molecular aberrations, including DNA methylation and gene mutations, are involved in gastric carcinogenesis. Interestingly, the frequency of genetic aberrations differs among epigenotypes (Table 2) [23]. E-HME is uniquely observed in $\mathrm{EBV}(+) \mathrm{GCs}$. $\mathrm{EBV}(+)$ GCs histologically demonstrate poorly differentiated carcinoma with dense infiltration of lymphocytes, referred to as gastric carcinoma with lymphoid stroma [4]. EBV(+) GCs frequently have $C D K N 2 A$ silencing by DNA methylation. PIK3CA mutations, $P D-L 1 / 2$ overexpression, and lack of TP53 mutations are frequently observed in this epigenotype (Fig. 2) [3, 23]. MSI, which is categorized as HME, often has $M L H 1$ silencing by DNA methylation. Although the MSI subtype generally lacks targetable amplifications, MSI has abundant gene mutations, including PIK3CA, $A R I D 1 A$, and RTK. MSI also frequently has a mutation of histocompatibility complex class I genes, including $B 2 M$ and $H L A-B$, which may work better in hypermutated GCs by suppressing the immune response [23]. LME contains GS and CIN subtypes. GS subtype is generally of diffuse type and frequently has CLDN18-ARHGAP fusion or CDH1/RHOA mutations [3]. The CIN subtype, which has genomic amplification of RTKs, is generally of intestinal type and frequently has TP53 mutations [23].

\section{Conclusion}

DNA methylation in the $\mathrm{CpG}$ island is associated with gastric carcinogenesis. GCs are classified into 3 epigenotypes by DNA methylation. In addition to DNA methylation, variable genetic aberrations are involved in gastric carcinogenesis. Interestingly, the frequency and targets of genetic aberrations vary depending on the epigenotype. Aberrations in the genome and epigenome are expected to synergistically interact and contribute to gastric carcinogenesis, and comprehensive analyses of those in GCs may help elucidate the mechanism of carcinogenesis.

\section{Conflict of Interest Statement}

The authors have no conflict of interest to disclose.

\section{Funding Sources}

Grants $19 \mathrm{H} 03726$ and 20K16166 from the Japan Society for the Promotion of Science; grant 19cm0106510h0004 and 19ck0106263h0003 from the Japan Agency for Medical Research and Development; grant 2018-Y9 from the Global and Prominent Research, Chiba University.

\section{Author Contributions}

G.U. and A.K. drafted the manuscript. K.M., Y.M., M.U., and S.F. revised the manuscript critically for important intellectual content. M.F., T.U., and A.K. supervised this review article.

\footnotetext{
References $\quad 1$ Torre LA, Bray F, Siegel RL, Ferlay J, LortetTieulent J, Jemal A. Global cancer statistics, 2012. CA Cancer J Clin. 2018;65(2):87-424.

2 Lauren P. The two histological main types of gastric carcinoma: diffuse and so-called intestinal-type carcinoma. An attempt at a histoclinical classification. Acta Pathol Microbiol Scand. 1965;64:31-49.

3 Tan P, Yeoh KG. Genetics and molecular pathogenesis of gastric adenocarcinoma. Gastroenterology. 2015;149(5):1153-62.
}

DNA Methylation and Genetic Aberrations in GC
Digestion 2021;102:25-32

DOI: $10.1159 / 000511243$ 
4 Carneiro F, Fukayama M, Grabsch HI, Yasui $\mathrm{W}$. WHO classification of tumours, digestive system tumours. In: Lokuhetty D, White VA, Watanabe R, Cree IA, editors. Gastric adenocarcinoma. 5th ed. Lyon, France: IARC Press; 2019. Vol. 1; p. 85-95.

5 Ueyama H, Yao T, Nakashima Y, Hirakawa K, Oshiro Y, Hirahashi M, et al. Gastric adenocarcinoma of fundic gland type (chief cell predominant type): proposal for a new entity of gastric adenocarcinoma. Am J Surg Pathol. 2010;34(5):609-19.

6 Uemura N, Okamoto S, Yamamoto S, Matsumura N, Yamaguchi S, Yamakido M, et al. Helicobacter pylori infection and the development of gastric cancer. N Engl J Med. 2001; 345(11):784-9.

7 Matsusaka K, Funata S, Fukayama M, Kaneda A. DNA methylation in gastric cancer, related to Helicobacter pylori and Epstein-Barr virus. World J Gastroenterol. 2014;20(14):3916-26.

8 Feinberg AP, Tycko B. The history of cancer epigenetics. Nat Rev Cancer. 2004;4(2):14353.

9 Padmanabhan N, Ushijima T, Tan P. How to stomach an epigenetic insult: the gastric cancer epigenome. Nat Rev Gastroenterol Hepatol. 2017;14(8):467-78.

10 Toyota M, Ahuja N, Suzuki H, Itoh F, OheToyota M, Imai K, et al. Aberrant methylation in gastric cancer associated with the $\mathrm{CpG}$ island methylator phenotype. Cancer Res. 1999;59(21):5438-42.

11 Kaneda A, Kaminishi M, Yanagihara K, Sugimura T, Ushijima T. Identification of silencing of nine genes in human gastric cancers. Cancer Res. 2002;62(22):6645-50.

12 Ushiku T, Chong JM, Uozaki H, Hino R, Chang MS, Sudo M, et al. p73 gene promoter methylation in Epstein-Barr virus-associated gastric carcinoma. Int J Cancer. 2007;120(1): 60-6.

13 Matsusaka K, Kaneda A, Nagae G, Ushiku T, Kikuchi Y, Hino R, et al. Classification of Epstein-Barr virus-positive gastric cancers by definition of DNA methylation epigenotypes. Cancer Res. 2011;71(23):7187-97.

14 Flavahan WA, Drier Y, Liau BB, Gillespie SM, Venteicher AS, Stemmer-Rachamimov AO, et al. Insulator dysfunction and oncogene activation in IDH mutant gliomas. Nature. 2016;529(7584):110-4.

15 Hnisz D, Weintraub AS, Day DS, Valton AL, Bak RO, Li CH, et al. Activation of proto-oncogenes by disruption of chromosome neighborhoods. Science. 2016;351(6280):1454-8.

16 Ooi WF, Nargund AM, Lim KJ, Zhang S, Xing $\mathrm{M}$, Mandoli A, et al. Integrated paired-end enhancer profiling and whole-genome sequencing reveals recurrent CCNE1 and IGF2 enhancer hijacking in primary gastric adenocarcinoma. Gut. 2020;69(6):1039-52.
17 Okabe A, Huang KK, Matsusaka K, Fukuyo $\mathrm{M}$, Xing $\mathrm{M}$, Ong $\mathrm{X}$, et al. Cross species chromatin interactions drive transcriptional rewiring in Epstein Barr virus positive gastric adenocarcinoma. Nat Genet. 2020;52(9):91930.

18 Vogelstein B, Papadopoulos N, Velculescu VE, Zhou S, Diaz LA Jr, Kinzler KW. Cancer genome landscapes. Science. 2013;339(6127): 1546-58.

19 Hanazono K, Natsugoe S, Stein HJ, Aikou T, Hoefler H, Siewert JR. Distribution of p53 mutations in esophageal and gastric carcinomas and the relationship with p53 expression. Oncol Rep. 2006;15(4):821-4.

20 Wang K, Kan J, Yuen ST, Shi ST, Chu KM, Law S, et al. Exome sequencing identifies frequent mutation of ARID1A in molecular subtypes of gastric cancer. Nat Genet. 2011; 43(12):1219-23.

21 Wang K, Yuen ST, Xu J, Lee SP, Yan HH, Shi ST, et al. Whole-genome sequencing and comprehensive molecular profiling identify new driver mutations in gastric cancer. Nat Genet. 2014;46(6):573-82.

22 Kakiuchi M, Nishizawa T, Ueda H, Gotoh K, Tanaka A, Hayashi A, et al. Recurrent gain-offunction mutations of RHOA in diffuse-type gastric carcinoma. Nat Genet. 2014;46(6): 583-7.

23 Cancer Genome Atlas Research Network. Comprehensive molecular characterization of gastric adenocarcinoma. Nature. 2014; 513(7517):202-9.

24 Christensen BC, Houseman EA, Marsit CJ, Zheng S, Wrensch MR, Wiemels JL, et al. Aging and environmental exposures alter tissuespecific DNA methylation dependent upon CpG island context. PLoS Genet. 2009;5(8): e1000602.

25 Katsurano M, Niwa T, Yasui Y, Shigematsu Y, Yamashita S, Takeshima H, et al. Early-stage formation of an epigenetic field defect in a mouse colitis model, and non-essential roles of T- and B-cells in DNA methylation induction. Oncogene. 2012;31(3):342-51.

26 Niwa T, Tsukamoto T, Toyoda T, Mori A, Tanaka $\mathrm{H}$, Maekita $\mathrm{T}$, et al. Inflammatory processes triggered by Helicobacter pylori infection cause aberrant DNA methylation in gastric epithelial cells. Cancer Res. 2010; 70(4):1430-40.

27 Niwa T, Toyoda T, Tsukamoto T, Mori A, Tatematsu M, Ushijima T. Prevention of Helicobacter pylori-induced gastric cancers in gerbils by a DNA demethylating agent. Cancer Prev Res. 2013;6(4):263-70.

28 Kaneda A, Matsusaka K, Aburatani H, Fukayama $M$. Epstein-Barr virus infection as an epigenetic driver of tumorigenesis. Cancer Res. 2012;72(14):3445-50.
29 Matsusaka K, Funata S, Fukuyo M, Seto Y, Aburatani H, Fukayama M, et al. Epstein-Barr virus infection induces genome-wide de novo DNA methylation in non-neoplastic gastric epithelial cells. J Pathol. 2017;242(4):391-9.

30 Namba-Fukuyo H, Funata S, Matsusaka K, Fukuyo M, Rahmutulla B, Mano Y, et al. TET2 functions as a resistance factor against DNA methylation acquisition during Epstein-Barr virus infection. Oncotarget. 2016; 7(49):81512-26.

31 Okabe A, Funata S, Matsusaka K, Namba H, Fukuyo M, Rahmutulla B, et al. Regulation of tumour related genes by dynamic epigenetic alteration at enhancer regions in gastric epithelial cells infected by Epstein-Barr virus. Sci Rep. 2017;7(1):7924.

32 Asakawa Y, Okabe A, Fukuyo M, Li W, Ikeda E, Mano Y, et al. Epstein-Barr virus-positive gastric cancer involves enhancer activation through activating transcription factor 3 . Cancer Sci. 2020;111(5):1818-28.

33 Sakatani T, Kaneda A, Iacobuzio-Donahue CA, Carter MG, de Boom Witzel S, Okano H, et al. Loss of imprinting of Igf2 alters intestinal maturation and tumorigenesis in mice. Science. 2005;307(5717):1976-8.

34 Kaneda A, Feinberg AP. Loss of imprinting of IGF2: a common epigenetic modifier of intestinal tumor risk. Cancer Res. 2005;65(24): 11236-40.

35 Maekita T, Nakazawa K, Mihara M, Nakajima T, Yanaoka K, Iguchi M, et al. High levels of aberrant DNA methylation in Helicobacter pylori-infected gastric mucosae and its possible association with gastric cancer risk. Clin Cancer Res. 2006;12(3 Pt 1):989-95.

36 Yamashita S, Kishino T, Takahashi T, Shimazu T, Charvat H, Kakugawa Y, et al. Genetic and epigenetic alterations in normal tissues have differential impacts on cancer risk among tissues. Proc Natl Acad Sci U S A. 2018;115(6):1328-33.

37 Asada K, Nakajima T, Shimazu T, Yamamichi N, Maekita T, Yokoi C, et al. Demonstration of the usefulness of epigenetic cancer risk prediction by a multicentre prospective cohort study. Gut. 2015;64(3):388-96.

38 Maeda M, Nakajima T, Oda I, Shimazu T, Yamamichi N, Maekita T, et al. High impact of methylation accumulation on metachronous gastric cancer: 5-year follow-up of a multicentre prospective cohort study. Gut. 2017;66(9): 1721-3.

39 Funata S, Matsusaka K, Yamanaka R, Yamamoto S, Okabe A, Fukuyo M, et al. Histone modification alteration coordinated with acquisition of promoter DNA methylation during Epstein-Barr virus infection. Oncotarget. 2017;8(33):55265-79.

40 Grabsch HI, Tan P. Gastric cancer pathology and underlying molecular mechanisms. Dig Surg. 2013;30(2):150-8. 